\title{
Monitoring a municipal wastewater treatment process using a trend analysis
}

Tomperi Jani $^{\mathrm{a}}$, Juuso Esko ${ }^{\mathrm{a}}$, Kuokkanen Anna ${ }^{\mathrm{b}}$, Leiviskä Kauko ${ }^{\mathrm{a}}$

${ }^{a}$ Control Engineering, University of Oulu, Oulu, Finland.

${ }^{\mathrm{b}}$ HSY Helsinki Region Environmental Services Authority, Helsinki, Finland

Corresponding author: e-mail jani.tomperi@oulu.fi. University of Oulu, Control Engineering, P.O. Box 4300, FI-90014 University of Oulu, Finland.

\begin{abstract}
New monitoring methods are required to enhance the operation of a wastewater treatment process and to meet the constantly tightening regulations for the effluent discharges. An on-line optical monitoring device, that analyses the morphological parameters of the flocs, have been showed to a potential tool for assessing the wastewater quality and the state of the activated sludge process. In this paper, the earlier presented trend analysis method is applied to the operating conditions, the treatment results and the optical monitoring variables of a full-scale biological wastewater treatment process. The trend episodes and the deviation indices resulted from the trend analysis provide warning of the changes in the monitored variables and the received information can be used as assistance in the treatment process operation and avoiding harmful environmental risks.
\end{abstract}

Keywords: Activated sludge; Optical monitoring; Morphology; Nonlinear; Operation conditions;

\section{Introduction}

Wastewater treatment should efficiently remove oxygen demanding substances, excessive nutrients and toxicants from the wastewater. Wastewater treatment in active sludge processes (ASPs), which are 
commonly used for treating wastewaters, is based on the growth and activity of micro-organisms that consume the organic matter from the wastewater and enable the development of flocs. In ASP, several physical, chemical and microbiological mechanisms simultaneously affect the purification result but the efficient treatment process is highly dependent on the condition of the biomass. Complex nonlinear ASPs are very sensitive to rapid and extreme external and internal disturbances, for example changing temperature and the varying quality and quantity of the influent wastewater, that affect the bacterial balance in the biomass. Disturbances in the bacterial balance will most often be shown as dysfunctional flocculation which results low effluent quality and thus environmental problems. The most common problems in an ASP are pinpoint flocs formation, dispersed growth, viscous (zoogleal) bulking and filamentous bulking which is caused when the biomass is strongly colonized by long filaments that hold the flocs apart and hinder the sludge settling in the secondary settling tank causing the increase of the amount of total suspended solids content in the effluent and the reduction in the quality of treated wastewater. Recovering from the disturbances is slow and thus the effects on process operation and purification result are long-lasting. [1-3]

To avoid serious problems or just to meet the constantly tightening limitations to the effluent discharges defined by the authorities and keeping the operation costs at minimum, an early detection of changes in the operating conditions and treatment results is highly important as the accurate monitoring is the basis of the reliable process control. Although the expert knowledge and traditional offline analysis of wastewater samples are important in process operation, there is a demand for new on-line monitoring tools and methods to add the knowledge about the state of the process and to improve the process operation. Temporal reasoning based on trend information is a valuable tool for the monitoring of slow processes. Detecting changes is important for process control and fault diagnosis. Typical reasoning systems have a language to represent the trends, a technique to identify the trends, and a 
mapping from the trends to operational conditions [4]. The fundamental elements are modelled geometrically as triangles to describe local temporal patterns in data (Figure 1). The elements, triangular episodic representations [5] are defined by the signs of the first and second derivatives, respectively. Linear regression combined with fuzzy reasoning can be used in detecting significant changes up or down in process variables [6]. The qualitative scaling defined by episodes shown in Figure 1 generates a hierarchical multiscale structure of trend descriptions over different time scales from process data [7].

In this paper, the earlier presented trend analysis method $[8,9]$ based on generalized norms $[10,11]$ and nonlinear scaling [12] is applied to a real long-period data of a full-scale municipal wastewater treatment process. The objective is to receive warnings of the changes in the inspected variables using the trend analysis method and to demonstrate the functionality of the developed method in multifold processes as it was earlier utilized for instance to provide the early detection of changes in a water treatment process [13], in condition monitoring [14] and a solar thermal power plant [15]. At first, the nonlinear scaling and trend analysis methods are briefly introduced and then utilized to find the trend episodes and deviation indices for the operating conditions, the treatment results and the on-line optical monitoring results, which were carried out using an optical monitoring device designed especially for in-situ operation and presented in [16]. The calculated trend episodes and deviation indices show the direction, speed and the severity of the change in the observed variable. Thus, a warning of the change is provided and the received information can be used as assistance in operating the process and avoiding harmful environmental risks.

\section{Material and Methods}

\subsection{Wastewater treatment plant}


The research site was located in Helsinki Viikinmäki. The largest wastewater treatment plant (WWTP) in Finland build inside the bedrock processes the wastewaters of over 800,000 inhabitants and the nearby industrial sources with the average daily flow of about $270,000 \mathrm{~m}^{3}$ of wastewater but melting snow and hard rains often multiply the flow. The WWTP is a three-phased activated sludge process that utilizes the simultaneous precipitation method for phosphorus removal. Wastewater is processed in nine parallel activated sludge process lines. In addition to mechanical, biological and chemical treatment, a biological filter has been added to improve the nitrogen removal. The process removes $95 \%$ of the phosphorus and $90 \%$ of the nitrogen together with nearly all solids and oxygen-consuming substances from the wastewater. The biological treatment is conducted by means of a de-nitrificationnitrification process in an aeration tank which is used to grow the activated sludge. The unit operations, the location of the on-line optical monitoring device and average operation delays of the process are shown in Figure 2. [17]

\subsection{Optical monitoring and image analysis}

The traditional analyses of sampled wastewater do not provide fundamental knowledge on the wastewater quality or the essential information about the reasons for the problems in the treatment process. On-line optical monitoring of floc morphological characteristics, on the other hand, gives fast objective information about the state of the wastewater treatment process and reveals some of the reasons for settling problems $[3,18,19,20,21]$. The optical monitoring data used in this study was collected by a small-scale optical monitoring device which was especially designed for in-situ use to replace the slow, irregular and subjective manual microscopic analysis of wastewater samples [16]. The developed device and analysis methods were proved functional for monitoring the floc morphology reliably in-situ in the full-scale municipal ASP during a period of several consecutive months [18,21]. The optical monitoring device measured and analysed several morphological features of the flocs and 
filaments as for example the size parameters mean equivalent diameter, floc area and filament length, and the shape parameters fractal dimension, form factor and roundness. The parameters were calculated as an average of the values for individual objects over a single image. Monitoring device, image processing and analyses methods and the mathematical formulas of the calculated size and shape parameters used in this study are presented in detail in [16].

\subsection{Nonlinear scaling}

Before the below described trend analysis can be carried out the original data have to be scaled between $[-2,2]$ using the nonlinear scaling method based on generalized moments, norms and skewness presented earlier in [12]. A nonlinear mapping function was developed to extract the meanings of variables from measurement signals. These functions are called membership definitions which map the real values of variables to the linguistic range of $[-2,2]$. Thus, a normal scaling to range $[-1,1]$ is combined with the handling of warnings and alarms. A trapezoidal membership function which is based on the support and core areas $\left[c_{l}, c_{h}\right]$ is used to define the concept of the feasible range. The support area is defined by the minimum and maximum of the values of the variable $x$. The value range is divided into two parts by the central tendency value $c$ and the core area is limited by the central tendency values of the lower and upper part. The tuning approach based on the generalized skewness is used for estimating the central tendency value and the core area. The central tendency value is chosen by the point where the skewness changes from positive to negative. The dataset is divided into a lower part and an upper part and the same analysis is done for these two datasets. The estimates of the corner points are the points where the direction of the skewness changes for the lower and upper data set, respectively. The iteration is performed with generalized norms. The mapping function contains one monotonously increasing function for the values between -2 to 0 and one monotonously increasing function between values 0 to +2 . Membership functions consist of two second order polynomials: one 
for the negative values and one for the positive values. Because the scaling idea is based on the membership functions of fuzzy set systems these values are called linguistic values. The coefficients of the polynomials are defined by the corner points. The main concepts are presented in Figure 3, which shows the connection between the membership definition and the corresponding fuzzy membership functions.

\subsection{Trend analysis}

In the trend analysis applied, for any variable $x_{j}$ a trend index $I_{j}^{T}(k)$ is calculated from the scaled values $X_{j}$ based on the means obtained for a short and a long time periods, defined by window lengths $n_{S}$ and $n_{L}$, respectively. The time periods $n_{L}$ and $n_{S}$ are used for tuning the trend analysis to applications and fine-tuning can be done by adjusting the weight factors $w_{1}$ and $w_{2}$ used for the indices $I_{j}^{T}(k)$ and $\Delta I_{j}^{T}(k)$. The derivative of the trend index, $\Delta I_{j}^{T}(k)$, is used for analyzing triangular episodic representations. The trends are linear if the derivative is close to zero. An increase is detected if the trend index exceeds a threshold and correspondingly, if the trend index is below a threshold, a decrease is detected. The index value in range $[-2,2]$ represents the strength of both decrease and increase of the variable $x_{j}$ [23]. A concave upward monotonic increase (D) and a concave downward monotonic decrease (B) are dangerous situations where situation is bad and it is getting worse with increasing speed (Figure 1). A concave downward monotonic increase (A) and a concave upward monotonic decrease $(\mathrm{C})$ mean that an unfavorable trend is stopping. The need for changing the operation strategy depends on the severity of the situation, which is evaluated by a deviation index $I_{j}^{D}(k)$. Deviation index has its highest absolute values, when the difference to the set point is very large and is getting still larger with a fast increasing speed. [9] 
The time periods and the weight factors are application specific. The length of the time window in the trend analysis depends on the process and the purpose of the analysis. The long time windows are usually applied in monitoring the process and short time windows are more suitable in control, where faster reacting is demanded. Weight factors and preset parameters affect the accuracy of the trend analysis results and they are selected separately for every measurement. In this study, weight factors and window lengths were selected for monitoring purposes: the short and long windows were 5 and 20 measurements, respectively. In the process monitoring, the concave upward monotonic increase (D) and concave downward monotonic decrease (B) require special attention. New phenomena are activated when the system moves from the linear increase to this area. The trend episodes are visualized step by step during the operation but the sequence of the points is not seen when all the points are presented in the plotted still figures, as in the following.

\section{Results and discussion}

The described trend analysis method was utilized to monitor the full-scale municipal wastewater treatment process and to provide the warnings of the changes. Trend analysis was applied to the selected variables considered important from the dataset which consisted of the on-line optical monitoring results and the traditional wastewater treatment process measurements from a period of over one year. The on-line optical monitoring measurements were carried out at least once a day but some of the process measurements, on the other hand, were done only two to three times a week. To avoid approximations and misleading results, the missing data were not interpolated. In addition, during the process maintenance stoppages measurements could not be performed. Thus, the total amount of data was 97 usable measurement times. Before performing the trend analysis, all data were scaled between $[-2,2]$. Scaled values can be plotted to a control chart where horizontal lines indicate 
limits for operational purposes. In bidirectional measurements, limits of three kind can be presented: upper and lower warning limits, upper and lower alarm or action limits, and upper and lower specification limits. Warning limits do not require immediate actions but if the value exceeds an alarm limit, control actions are needed to restore the process to the stable condition. Outside the specification limits the data point is out of the specification. Warning and alarm limits can be considered as early warning systems so that problems can be solved before the values outside the acceptable specification limits occur. The control chart of one-directional measurements can be divided into three or four parts using horizontal limit lines (excellent, normal, bad and dangerous situation, for example).

\subsection{The operating conditions and controllable variables}

The operating conditions monitoring is aimed to provide warnings about the changes which may affect the operation of the wastewater treatment process. The optimal range of each operating condition is plant and process specific. In this paper, the studied operating conditions are the temperature and influent flow which are not controllable variables and thus can vary significantly. The study of other operation conditions is not presented.

The sludge age and sludge concentration, on the other hand, are controllable. Suitable sludge age and sludge concentration are maintained by removing part of the activated sludge regularly from the treatment process. The sludge concentration depends on the influent load and the sludge age. Incoming load is again partly dependent on the flow rate and the season of the year. The sludge age is also dependent on the season of the year because to ensure nitrification throughout the year it is controlled mainly based on wastewater temperature. The sludge age is one of the factors that determine which bacterial groups are dominant in the activated sludge and how the bacteria grow and form flocs. For 
example, if the sludge age is too high related to the temperature, the amount of filaments may increase which hinders the settleability and bulking may occur.

As presented earlier in [21], in the municipal WWTP the temperature and flow has a mutual correlation and based on the correlation analysis when the flow was high several variables measured from the incoming wastewater were at the low level due to the diluting effect of rainfall and melting waters. The wastewater temperature correlated with the effluent biological oxygen demand (BOD) and suspended solids (SS) and also with several image analysis variables. For example, when the temperature was high in the summer, the level of SS and BOD were low, and the filament length, amount of filaments and number of measured objects were low, and the roundness of the flocs was high.

In Figure 4, the scaled values and the trend analysis results of the influent flow and the wastewater temperature are presented in order from the top: the scaled measurement values, the deviation index, the trend episodes and trend indices which clarify the behaviour of the trend episodes. For the efficient treatment process in a municipal WWTP, the flow should be constant and the temperature should be at the normal or high level. Here, the temperature rose linearly, was at the very high level at the end of the summer and decreased to the lowest level fast and with increasing speed. The rapid decrease is shown in the trend episode chart and in the deviation index as a warning. The flow varied through the year mostly due to the snow melting and heavy rains and it was at the highest level at the end of the period which is shown as an alarming situation in the deviation index. Few warning events are also detected during the period. The trend episode chart shows three separate situations when the flow rose with fast increasing speed and returned through the linear increase to constant and decreasing states. 
In Figure 5, the scaled values and the trend analysis results of sludge age and sludge concentration are presented in order from the top: the scaled measurement values, the deviation index, the trend episodes and trend indices. Sludge age was kept at low and normal level over half of the period but a fast rise with increasing speed was detected at the end of the summer. This could be considered as a dangerous change. The rise continued as a linear increase towards the end of the period and the sludge age was at the highest value in the end of the period. The fast rise to the high level is not alarming, but considered as warning as seen in the deviation index. At the same time the temperature was at the lowest level which supposedly affected the biomass and the quality of treated wastewater which required operational actions to the sludge age. Sludge concentration on the other hand followed the changes of the temperature and was at first on the high level but at the beginning of the summer it decreased fast and with increasing speed towards minimum. This is shown as alarming situation in the deviation index. After the decrease, the sludge concentration was in a constant state and started a slow linear increase.

\subsection{The treatment results}

The treatment efficiency of the WWTP can be assessed for example by the reduction of nutrients (nitrogen and phosphorus), suspended solids, biological oxygen demand and chemical oxygen demand (COD) measured from the treated wastewater. Here, only the BOD and suspended solids are studied as for the example. The level of SS and BOD in biologically treated wastewater followed the changes of the temperature: both SS and BOD were low in the summer when wastewater was warmer and rose when the temperature decreased. At the beginning of the period, SS was high and although changes (decrease) in both BOD and SS were fast the severity of situations was not alarming. On the contrary, the decrease of suspended solids and BOD show that the treatment process was efficient. The behaviour of BOD was more sedate than the behaviour of suspended solids even though some variation 
was detected. The rise of SS to the highest level before the summer was fast and triggered a warning but the second increase at the end of the period was more sedate and linear.

\subsection{On-line optical monitoring}

The wastewater samples for the on-line imaging were taken from the aeration tank of one activated sludge line. Here, two variables of the on-line optical monitoring, fractal dimension and roundness, are presented. Roundness is defined as the ratio between the area of an object and the area of a circle with a diameter equal to the object's length. The floc structure is described using the fractal dimension which is a measure of the irregularity of the perimeter.

The optical monitoring parameters have several mutual correlations, for example when the roundness of flocs was low the amount and length of filaments, and the number of small objects were high [20,21]. Roundness and fractal dimension have a very high negative mutual correlation coefficient. When the quality of the biologically treated wastewater was good and the temperature was high, the roundness of flocs was high and the fractal dimension was low. When the quality of the treated wastewater deteriorated the roundness of the flocs decreased and fractal dimension increased.

In Figure 7, the scaled values and the trend analysis results of fractal dimension and roundness are presented in order from the top: the scaled measurement values, the deviation index, trend episodes and trend indices. At the beginning of the period, the fractal dimension of flocs was very high. The decrease to minimum was sedate but the increase that started at the end of the summer was fast with increasing speed and reached the high level at the end of the period. This is shown as a dangerous situation in the trend episode chart and as a warning in the deviation index. The behaviour of the roundness of flocs was opposite to the fractal dimension. Roundness was at low level at the beginning of the research 
period, but increased to the high level during the summer. The fast decrease with increasing speed is shown as a dangerous situation in the trend episode chart and as a warning in the deviation index.

\section{Conclusions}

The accurate monitoring and control of a wastewater treatment process are important to meet the constantly tightening limitations to effluent discharges and to keep the operation costs at minimum. In addition to traditional process measurements, new monitoring methods and devices are required to receive more fundamental information on the treatment process and the quality of the wastewater. For the characterization of floc morphology an on-line optical monitoring device is a potential tool for receiving fast objective information about the quality of wastewater and to reveal some of the reasons for the problems in the process.

In this research, the earlier presented and here briefly described trend analysis method was applied to find trend episodes and deviation indices for operating conditions, the treatment results and the on-line optical monitoring variables of the full-scale municipal activated sludge process. The study showed the functionality of the trend analysis method applied also for a full-scale biological wastewater treatment monitoring providing information on the changes. The trend episodes and deviation indices showed the direction, speed and the severity of the changes in the observed variables. The received information can be used assistance in controlling the process and avoiding harmful environmental risks.

\section{Acknowledgements}

This research was carried out as part of the Measurement, Monitoring and Environmental Efficiency Assessment (MMEA), the research program of CLEEN Ltd. - Cluster for Energy and Environment. 


\section{References}

[1] Tchobanoglous G, Burton FL, Stense HD. Wastewater Engineering: Treatment and Reuse. $4^{\text {th }}$ edition. McGraw-Hill. Metcalf \& Eddy Inc. New York. 2003.

[2] Amaral AL, Ferreira EC. Activated sludge monitoring of a wastewater treatment plant using image analysis and partial least squares regression. Analytica Chimica Acta. 2005;544:246-253.

[3] Mesquita DP, Dias O, Amaral AL, Ferreira EC. Monitoring of activated sludge settling ability through image analysis: validation on full-scale wastewater treatment plants. Bioprocess and Biosystems Engineering. 2009;32:361-367.

[4] Dash S, Rengaswamy R, Venkatasubramanian V. Fuzzy-logic based trend classification for fault diagnosis of chemical processes. Computers and Chemical Engineering. 2003;27:347-362.

[5] Cheung JT-Y, Stephanopoulos G. Representation of process trends - part I. A formal representation framework. Computers and Chemical Engineering. 1990;14(4/5):495-510.

[6] Poirier PJ, Meech JA. Using fuzzy logic for on-line trend analysis. In 2nd IEEE Conference on Control Applications, Vancouver, B.C. 1993. p. 83-86.

[7] Stephanopoulos G, Locher G, Duff MJ, Kamimura R. Fermentation database mining by pattern recognition. Biotechnology and Bioengineering. 1997;53(5):443-452.

[8] Juuso E, Latvala T, Laakso I. Intelligent analysers and dynamic simulation in a biological water treatment process. In I. Troch and F. Breitenecker editors. 6th Vienna Conference on Mathematical Modelling - MATHMOD 2009, February 11-13, 2009, Argesim Report no. 35, 2009. 999-1007.

[9] Juuso EK. Intelligent trend indices in detecting changes of operating conditions. In 2011 UKSim 13th International Conference on Modelling and Simulation, IEEE Computer Society, 30 March1 April 2011, Cambridge, UK. 2011.162-167. 
[10] Lahdelma S, Juuso E. Signal processing and feature extraction by using real order derivatives and generalised norms. Part 1: Methodology. The International Journal of Condition Monitoring. 2011;1(2):46-53.

[11] Lahdelma S, Juuso E. Signal processing and feature extraction by using real order derivatives and generalised norms. Part 2: Applications. The International Journal of Condition Monitoring. 2011;1(2):54-66.

[12] Juuso E, Lahdelma S. Intelligent scaling of features in fault diagnosis. Proceedings of 7th International Conference on Condition Monitoring and Machinery Failure Prevention Technologies, 22-24 June, 2010, Stratford-upon-Avon, UK. 2010.

[13] Tomperi J, Juuso E, Leiviskä K. Early warning of changing drinking water quality by trend analysis. Journal of Water and Health. 2016;14(3):433-442.

[14] Lahdelma S, Juuso E. Trend Analysis in Condition Monitoring of Process Equipments. $8^{\text {th }}$ International Conference on Condition Monitoring and Machinery Failure Prevention Technologies, CM 2011 / MFPT 2011, Cardiff, UK, 20-22 June 2011.

[15] Juuso EK, Yebra LJ. Optimisation of solar energy collection with smart adaptive control. Industrial Electronics Society, IECON 2013 - 39th Annual Conference of the IEEE. 10-13 Nov. 2013.

[16] Koivuranta E, Keskitalo J, Haapala A, Stoor T, Sarén M, Niinimäki J. Optical monitoring of activated sludge flocs in bulking and non-bulking conditions. Environmental Technology. 2013;34(5-8):679-686.

[17] HSY Viikinmäki wastewater treatment plant webpage. Available from: https://www.hsy.fi/en/experts/water-services/wastewater-treatmentplants/viikinmaki/Pages/default.aspx, 2017 (cited February 2017) 
[18] Koivuranta E, Stoor T, Hattuniemi J, Niinimäki J. On-line optical monitoring of activated sludge floc morphology. Journal of Water Process Engineering. 2015;5:28-34.

[19] Banadda EN, Smets IY, Jenne R, Van Impe JF. Predicting the onset of filamentous bulking in biological wastewater treatment systems by exploiting image analysis information. Bioprocess and Biosystems Engineering. 2005;27:339-348.

[20] Tomperi J, Koivuranta E, Kuokkanen A, Juuso E, Leiviskä K. Real-time optical monitoring of the wastewater treatment process. Environmental Technology. 2016;37(3):344-351.

[21] Tomperi J, Koivuranta E, Kuokkanen A, Leiviskä K. Modelling the effluent quality based on a real-time optical monitoring of the wastewater treatment process. Environmental Technology. 2017;38(1):1-13.

[22] Juuso EK. Integration of Intelligent Systems in Development of Smart Adaptive Systems. International Journal of Approximate Reasoning. 2004;35(3):307-337.

[23] Juuso EK. Tuning of large-scale linguistic equation (LE) models with genetic algorithms. In: Kolehmainen M., editor. Revised selected papers of the International Conference on Adaptive and Natural Computing Algorithms - ICANNGA 2009, Kuopio, Finland. Lecture Notes in Computer Science 5495, Heidelberg: Springer-Verlag, LNCS; 2009. p. 161-170. 
Figure captions:

Figure 1. Triangular episodic representations defined by the trend index $I_{j}^{T}(k)$ and the derivative of the index $\Delta I_{j}^{T}(k)$. Seven types of episodes are used for interval description. Redesigned from [5,9].

Figure 2. Wastewater treatment process at Viikinmäki WWTP. Modified from [17].

Figure 3. (A) The feasible range, (B) scaled value, and (C) membership functions. Redesigned from $[22]$.

Figure 4. The scaled values, the deviation indices, trend episodes, and trend indices and derivatives of the trend indices of flow (left) and temperature of wastewater (right).

Figure 5. The scaled values, the deviation indices, trend episodes, and trend indices and derivatives of the trend indices of sludge age (left) and sludge concentration (right).

Figure 6. The scaled values, the deviation indices, trend episodes, and trend indices and derivatives of the trend indices of BOD (left) and suspended solids (right) in biologically treated wastewater.

Figure 7. The scaled values, the deviation indices, trend episodes, and trend indices and derivatives of the trend indices of fractal dimension (left) and roundness of flocs (right). 